\title{
Static Schwarzschild Affine Connection Revised by Physical Decomposition of Gravitational Field
}

\author{
Ben-Chao Zhu \\ Department of physics and mathematics, Hubei \\ University of Medicine, shiyan, China
}

Jia Guo

Department of physics and mathematics, Hubei University of Medicine, shiyan, China

\author{
Guang-Xiao He \\ Department of physics and mathematics, Hubei \\ University of Medcine, shiyan, China \\ Peng-Cheng Zhang* \\ Department of physics and mathematics, Hubei \\ University of Medicine, shiyan, China \\ e-mail: pengchengzhang@163.com
}

*/corresponding author

\begin{abstract}
Physical decomposition of gauge fields and gravitational field are interesting subjects for many researchers $[1,2,3,4]$. The decomposition of gauge field in Refs $[1,2,3]$ has successfully solved the two-decade gauge problem of a meaningful gluon spin. Ref[4] extended this method to the gravitational field and attacked the longstanding problem of gravitational energy density. The metric is unambiguously separated into a pure geometric term which contributes null curvature tensor, and a physical term which represents the true gravitational effect and always vanishes in a flat space-time. In this paper, we will find the static Schwarzschild solution in the physical decomposition of gravitational field. Moreover, we compare the above solution with the ordinary static Schwarzschild solution . The differences between them show that there perhaps exist some unknown gravitational field energy out of static gravitational source. This conclusion probably help us to find the graviton from the deep universe by the modern advanced doctors.
\end{abstract}

Keywords- physical decomposition; gauge field; gravitational field; affine connection; static Schwarzschild

\section{INTRODUCTION}

During the last a few years, Chen and his co-workers have done some amazing work in decomposing gauge fields (including Abelian and non-Abelian fields) into gauge independent part (which they called physical part) and gauge dependent part (which carry all the gauge degrees of freedom [1,2]. The essential work in their papers in Refs.[1,2] is decomposing the gauge field like below

$$
A_{m}=\hat{A}_{m}+\bar{A}_{m}
$$

$\hat{A}_{\mu}$ is a physical term which is gauge-covariant and always vanishes in the vacuum, and the $\bar{A}_{\mu}$ is a puregauge term which solely carries (particularly, it does not contribute to electric or magnetic field strength), when we replace $A_{\mu}$ with $\hat{A}_{\mu}$ and the ordinary derivative with the pure-gauge covariant derivative constructed with $\bar{A}_{\mu}$ instead of $A_{\mu}$, we will surely reduce a gauge-dependent quantity to be a gauge-independent, such as the gluon spin $S=E \times A$. In their papers, they also proofed the puregauge term $\bar{A}_{\mu}$ completely carries all gauge-dependent freedoms (so that the quantity constructed by physical term $\hat{A}_{\mu}$ and pure-gauge covariant derivative constructed with $\bar{A}_{\mu} \quad$ is absolutely gauge-independent [1]). The mathematical way to separate $A_{\mu}$ to $\hat{A}_{\mu}$ can be expressed as :[1,2]

$$
\begin{gathered}
\hat{A}_{i}={\text { 抖 } j_{j}{ }_{k}^{-2} F_{j i}}^{\hat{A}_{0}=\underset{¥}{\mathrm{O}} d x^{i}\left(?_{0} \hat{A}_{i} \quad F_{i 0}\right)}
\end{gathered}
$$

In Eq. (3), the index $i$ takes any value of 1 to 3, and is not summed, and $\hat{A}_{i}$ is to be substituted by the expression in Eq. (3).

The Einstein's gravitational theory has a deep analogy with the non-Abelian gauge theory. The gauge invariance of gravitational theory refers to general covariance under arbitrary coordinate transformation. In gravitational theory, the covariant Riemann curvature tensor, $R_{\sigma \mu v}^{\rho}$, which is a true tensor and always zero in an intrinsically flat spacetime, is built out of the non-covariant connection

$$
R_{s m n}^{r}=\text { 禛 }{ }_{s n}^{r} \text { - 禛 }{ }_{s m}^{r}+\mathrm{G}_{b m}^{r} \mathrm{G}_{s n}^{b}-\mathrm{G}_{b n}^{r} \mathrm{G}_{s m}^{b}
$$

On the other hand, the non-tonsorial quantity affine connection $\Gamma_{\sigma \mu}^{\rho}$ carries all gauge term in coordinate transformation $x^{\mu} \rightarrow x^{\prime \mu}$ 


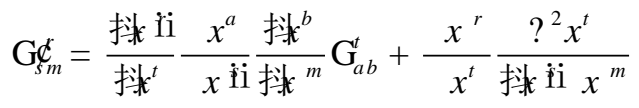

The affine connection $\Gamma_{\sigma \mu}^{\rho}$ can be made non-zero in an intrinsically flat space-time by using curvilinear coordinates, or eliminated away at any given point in an intrinsically curved space-time by choosing a suitable noninertial reference frame. This property is the reason why gravitational energy density in the traditional pseudotensor constructions meaningless.

\section{PHYSICAL DECOMPOSITION OF GRAVITATIONAL FIELD}

Analogous to the physical decomposition of the gauge field, we can write

$$
\Gamma_{\sigma \mu}^{\rho}=\bar{\Gamma}_{\sigma \mu}^{\rho}+\hat{\Gamma}_{\sigma \mu}^{\rho}
$$

the physical term $\hat{\Gamma}_{\sigma \mu}^{\rho}$ is always vanishes as does curved tensor $R_{\sigma \mu \nu}^{\rho}$ in an intrinsically flat space-time, the puregeometric term $\bar{\Gamma}_{\sigma \mu}^{\rho}$ gives zero curved tensor and solely carries gauge freedom in coordinates transformation. In Ref [4], we have demonstrated that the separation Eq. (6) has the same effect as the separation Eq. (1) in gauge fields. We can get the physical decomposition of gravitational field formula as following equations:

$$
\begin{aligned}
\bar{R}_{\sigma \mu v}^{\rho}= & \partial_{\mu} \bar{\Gamma}_{\sigma v}^{\rho}-\partial_{v} \bar{\Gamma}_{\sigma \mu}^{\rho} \\
& +\bar{\Gamma}_{\beta \mu}^{\rho} \bar{\Gamma}_{\sigma v}^{\beta}-\bar{\Gamma}_{\beta \nu}^{\rho} \bar{\Gamma}_{\sigma \mu}^{\beta} \\
= & 0 \\
\bar{D}_{i} \hat{\Gamma}_{\sigma i}^{\rho} & =\partial_{i} \hat{\Gamma}_{\sigma i}^{\rho}+\bar{\Gamma}_{i \alpha}^{\rho} \hat{\Gamma}_{\sigma i}^{\alpha} \\
& -\bar{\Gamma}_{i \sigma}^{\alpha} \hat{\Gamma}_{\alpha i}^{\rho}-\bar{\Gamma}_{i i}^{\alpha} \hat{\Gamma}_{\sigma \alpha}^{\rho} \\
& =0
\end{aligned}
$$

It means we can eliminate the spurious gravitational effect of the gravitational energy density by using the physical term $\hat{\Gamma}_{\sigma \mu}^{\rho}$ instead of $\Gamma_{\sigma \mu}^{\rho}$ and using the pure-gauge covariant derivatives instead of covariant derivatives. In Ref [4], we also presented the concrete procedure to compute a physically meaningful energy density of the gravitational field. For a finite and weak gravitational system, we can use vanishing boundary values, and apply perturbative expansions for both $\hat{\Gamma}_{\sigma \mu}^{\rho}$ and $\bar{\Gamma}_{\sigma \mu}^{\rho}$ :

$$
\begin{aligned}
\bar{\Gamma}_{\sigma i}^{\rho} & =\partial_{i}\left(\partial_{k} \partial_{k}\right)^{-1} \partial_{j} \Gamma_{\sigma j}^{\rho}+\text { highorder } \\
& =\partial_{i} \int_{-\infty}^{+\infty} \frac{\partial_{j} \Gamma_{\sigma j}^{\rho}}{4 \pi\left|x-x^{\prime}\right|} d^{3} x^{\prime}+\text { highorder } \\
\bar{\Gamma}_{\sigma 0}^{\rho} & =\int_{\infty}^{x} d x^{i} \partial_{0} \bar{\Gamma}_{\sigma i}^{\rho}+\text { highorder }
\end{aligned}
$$

The index $i$ in Eq. (9) takes any value from 1 to 3 , and is not summed. We have demonstrated that if a coordinate gives non-zero $\bar{\Gamma}_{\sigma \mu}^{\rho}$, it meant that it contains spurious gravitational effect, and one should use the physical affine connection $\hat{\Gamma}_{\sigma \mu}^{\rho}=\Gamma_{\sigma \mu}^{\rho}-\bar{\Gamma}_{\sigma \mu}^{\rho}$ to define gauge-independent quantities.

\section{THE REAL PHYSICAL ENERGY-MOMENTUM TENSOR OF GRAVITATIONAL FIELD}

How to get physical energy-momentum tensor of gravitational field? As first thought, it seems a simple exercise . One can follow what Einstein, Landau-Lifshitz, Møller and others had done to get energy-momentum "tensor" [6] (because of the non-tensor property of these quantities, here we use a quotation mark on tensor), which now are called traditional energy-momentum pseudotensors . In the position of our stand, the serious trouble of traditional pseudotensors is the metric and the affine connection all carry gauge freedom or spurious gravitational effect. So , one can not get the real physical energy-momentum tensor. Now, after physical decomposition of gravitational field can separate metric tensor and affine connection into physical part and nonphysical part, it is naturally to construct the real physical quantities by using the physical affine connection $\hat{\Gamma}_{\sigma \mu}^{\rho}=\Gamma_{\sigma \mu}^{\rho}-\bar{\Gamma}_{\sigma \mu}^{\rho}$, such as metric tensor $\hat{g}_{\mu v}$ and Ricci tensor $\hat{R}_{\mu v}$.What we need to do is to solve differential equation $\bar{g}_{\mu v}=\bar{g}(\bar{\Gamma})_{\text {(so that the }} \hat{g}_{\mu v}=g_{\mu v}-\bar{g}_{\mu v}$ ) with accordingly boundary condition $\bar{g}(\infty)$. If we obtain the physical metric tensor $\hat{g}_{\mu v}$, it is easy to obtain the true physical Ricci tensor $\hat{R}_{\mu \nu}$.

After illuminating how to obtain the real physical metric tensor $\hat{g}_{\mu v}$ and Ricci tensor $\hat{R}_{\mu v}$,we are now in the position to explain why the above technique we contrived can get the real physical energy-momentum tensor. From the Einstein equation with no cosmological constant $\Lambda$,

$$
R_{\mu v}-\frac{1}{2} g_{\mu v} R=8 \pi G T_{\mu v}
$$

By using the contracted Bianchi identity, we can get a native conclusion

$$
D_{\mu}\left(R_{\mu v}-\frac{1}{2} g_{\mu \nu} R\right)=8 \pi G D_{\mu} T_{\mu v}=0
$$

Here $D_{\mu} T_{\mu v}=0$ means that the general covariant energy-momentum conservation law of matter plus nongravitational field is still hold, but it makes no sense without gravitational field. If we separate metric tensor as $\hat{g}_{\mu v}+\bar{g}_{\mu v}$ and Ricci tensor as $\hat{R}_{\mu v}+\bar{R}_{\mu v}$, the Einstein equation can be reduced to

$$
\left(\bar{R}_{\mu v}+\hat{R}_{\mu v}\right)-\frac{1}{2}\left(\bar{g}_{\mu v}+\hat{g}_{\mu v}\right)(\bar{R}+\hat{R})=8 \pi G T_{\mu \nu}
$$




$$
\bar{R}_{\mu \nu}-\frac{1}{2} \bar{g}_{\mu \nu} \bar{R}=8 \pi G \tau_{\mu \nu}
$$

With $\tau_{\mu \nu}=T_{\mu \nu}+\frac{1}{8 \pi G} \hat{t}_{\mu \nu}$ and $\hat{t}_{\mu \nu}=\frac{1}{2} \hat{g}_{\mu \nu} \hat{R}-\hat{R}_{\mu \nu}$.

We can find that all quantities in Eq. (13) and Eq. (14) are pure-geometric terms. Then by using the pure-geometric background Bianchi identity, we can get

$$
\bar{D}_{\mu}\left(\bar{R}_{\mu \nu}-\frac{1}{2} \bar{g}_{\mu \nu} \bar{R}\right)=0
$$

the right hand side in Eq. (15) are non-linear terms which represent physical meaning of the system. If ${ }^{\bar{g}_{\mu v}}$ admits Killing vector $\bar{\xi}_{\mu}$, by replacing indices upstairs, the following ordinary differential conservation law can be achieved:

$$
\bar{D}_{\mu}\left(\tau^{\mu v}\right)=\partial_{\mu}\left(\tau^{\mu v} \bar{\xi}_{v}\right)=0
$$

$\tau^{\mu v} \bar{\xi}_{v}$ will give conserved four momentum :

$$
P^{\mu}=\int d^{3} x \tau^{\mu v} \bar{\xi}_{v}
$$

$\tau^{\mu \nu}$ is a true tensor and can be calculated by solving

$\bar{g}_{\mu v}=\bar{g}(\bar{\Gamma})$ with boundary condition .

Here, we need to briefly point out the differences of upgrading the energy-momentum tensor between what we have done and what Arnowitt-Deser-Misner(ADM) andAbbott-Deser(AD) had done previously. ADM scheme and $\mathrm{AD}$ scheme is to decompose metric ${ }^{g}{ }_{\mu \nu}$ into the sum of background ${ }^{g / o v}$ plus a whatever deviation $h_{\mu \nu}$, they also obtained the kind of results:

$$
\bar{D}_{\mu}\left(\tau^{\mu v}\right)=\partial_{\mu}\left(\tau^{\mu v} \bar{\xi}_{v}\right)=0
$$

whose explicit form is not needed here. But they just gave separating the whole metric to background and small deviation $_{\mu v}$. Finally, what is a real physical energymomentum tensor of gravitational field is not a question any more.

\section{SCHWARZSCHILD AFFINE CONNECTION IN PHYSICAL DECOMPOSITION OF GRAVITATIONAL FIELD}

As an explicated example, we will calculate the energy of Schwarzschild metric : the gravitational field outside a spherical, non-rotating mass. In the weak field approximation, the outside solution of Schwarzschild metric ( $r \geq R$ ) reads [7]

$$
d s^{2}=\left(1-\frac{2 m}{r}\right) d t^{2}-\left(1+\frac{2 m}{r}\right)\left(d x_{1}^{2}+d x_{2}^{2}+d x_{3}^{2}\right)
$$

The inner solution of Schwarzschild metric in the weak field approximation $(r \leq R)$ reads [7]:

$$
d s^{2}=\frac{1}{4 \pi}\left(1-\frac{r^{2}}{R^{2}}\right) d t^{2}-\left(1+\frac{r^{2}}{R^{2}}\right)^{-1}\left(d x_{1}^{2}+d x_{2}^{2}+d x_{3}^{2}\right)
$$

$R$ is the radius of matter. Then, it is a trivial work to calculate the outside nonzero affine connections,

$$
\begin{aligned}
& \Lambda_{01}^{0}=m x_{1}\left(\frac{1}{r^{3}}+\frac{2 m}{r^{4}}\right) \quad \Lambda_{02}^{0}=m x_{2}\left(\frac{1}{r^{3}}+\frac{2 m}{r^{4}}\right) \\
& \Lambda_{03}^{0}=m x_{3}\left(\frac{1}{r^{3}}+\frac{2 m}{r^{4}}\right) \quad \Lambda_{00}^{1}=m x_{1}\left(\frac{1}{r^{3}}-\frac{2 m}{r^{4}}\right) \\
& \Lambda_{00}^{3}=m x_{3}\left(\frac{1}{r^{3}}-\frac{2 m}{r^{4}}\right) \quad \Lambda_{00}^{2}=m x_{2}\left(\frac{1}{r^{3}}-\frac{2 m}{r^{4}}\right) \\
& \Lambda_{11}^{1}=\Lambda_{21}^{2}=\Lambda_{31}^{3}=-\Lambda_{22}^{1}=-\Lambda_{33}^{1}=m x_{1}\left(\frac{1}{r^{3}}-\frac{2 m}{r^{4}}\right) \\
& \Lambda_{22}^{2}=\Lambda_{12}^{1}=\Lambda_{22}^{3}=-\Lambda_{11}^{2}=-\Lambda_{33}^{2}=m x_{2}\left(\frac{1}{r^{3}}-\frac{2 m}{r^{4}}\right) \\
& \Lambda_{33}^{3}=\Lambda_{13}^{1}=\Lambda_{23}^{2}=-\Lambda_{11}^{3}=-\Lambda_{22}^{3}=m x_{3}\left(\frac{1}{r^{3}}-\frac{2 m}{r^{4}}\right)
\end{aligned}
$$

the inner nonzero affine connections are

$$
\begin{gathered}
\Upsilon_{01}^{0}=-x_{1}\left(\frac{1}{R^{2}}+\frac{r^{2}}{R^{4}}\right) \quad \Upsilon_{02}^{0}=-x_{2}\left(\frac{1}{R^{2}}+\frac{r^{2}}{R^{4}}\right) \\
\Upsilon_{03}^{0}=-x_{3}\left(\frac{1}{R^{2}}+\frac{r^{2}}{R^{4}}\right) \quad \Upsilon_{00}^{1}=-x_{1}\left(\frac{1}{R^{2}}+\frac{r^{2}}{R^{4}}\right) \\
\Upsilon_{00}^{2}=-x_{2}\left(\frac{1}{R^{2}}+\frac{r^{2}}{R^{4}}\right) \quad \Upsilon_{00}^{3}=-x_{3}\left(\frac{1}{R^{2}}+\frac{r^{2}}{R^{4}}\right) \\
\Upsilon_{11}^{1}=\Upsilon_{21}^{2}=\Upsilon_{31}^{3}=-\Upsilon_{22}^{1}=-\Upsilon_{33}^{1}=x_{1}\left(\frac{1}{R^{2}}-\frac{r^{2}}{R^{4}}\right) \\
\Upsilon_{22}^{2}=\Upsilon_{12}^{1}=\Upsilon_{32}^{3}=-\Upsilon_{11}^{2}=-\Upsilon_{33}^{2}=x_{2}\left(\frac{1}{R^{2}}-\frac{r^{2}}{R^{4}}\right) \\
\Upsilon_{33}^{3}=\Upsilon_{13}^{1}=\Upsilon_{23}^{2}=-\Upsilon_{11}^{3}=-\Upsilon_{22}^{3}=x_{3}\left(\frac{1}{R^{2}}-\frac{r^{2}}{R^{4}}\right)
\end{gathered}
$$

Then, Eq. (5) will be reduced to,

$$
\bar{\Gamma}_{\sigma i}^{\rho}=\frac{1}{4 \pi} \partial_{i}\left[\int_{0}^{x_{R}} \frac{\partial \Upsilon_{j \sigma j}^{\rho}}{\left|\bar{V}-x^{\prime}\right|} d^{3} x^{\prime}+\int_{x_{R}}^{+\infty} \frac{\partial_{j} \Lambda_{\sigma j}^{\rho}}{\left|x-x^{\prime}\right|} d^{3} x^{\prime}\right]
$$

The first term $\frac{1}{R^{2}}$ in Eqs. (27-32) do not contribute any result in Eqs. (33). So the pure-geometric term $\bar{\Gamma}_{\sigma \mu}^{\rho}$ in the far away from the matter $(r ? R)$ is 


$$
\begin{aligned}
\bar{\Gamma}_{\sigma i}^{\rho} & =\frac{1}{4 \pi} \partial_{i}\left\{\frac{1}{r}\left[\int_{0}^{x_{R}} \frac{3 r^{\prime 3}}{R^{4}} d^{3} x^{\prime}+\int_{x_{R}}^{+\infty} \frac{2 m^{2}}{r^{\prime}} d^{3} x^{\prime}\right]\right\} \\
& =\partial_{i}\left(\frac{\frac{1}{2} R^{2}+\frac{2 m^{2}}{R}}{r}\right)
\end{aligned}
$$

is a constant. Following what Einstein had done, we can get the non-physical affine connection are

$$
\begin{gathered}
\bar{\Gamma}_{11}^{1}=\bar{\Gamma}_{12}^{2}=\bar{\Gamma}_{13}^{3}=-\bar{\Gamma}_{22}^{1}=-\bar{\Gamma}_{33}^{1}=-4 m^{2} \frac{x_{1}}{r^{4}} \\
\bar{\Gamma}_{22}^{2}=\bar{\Gamma}_{23}^{3}=\bar{\Gamma}_{12}^{1}=-\bar{\Gamma}_{11}^{2}=-\bar{\Gamma}_{33}^{2}=-4 m^{2} \frac{x_{2}}{r^{4}} \\
\bar{\Gamma}_{33}^{3}=\bar{\Gamma}_{23}^{2}=\bar{\Gamma}_{13}^{1}=-\bar{\Gamma}_{11}^{3}=-\bar{\Gamma}_{22}^{3}=-4 m^{2} \frac{x_{3}}{r^{4}} \\
\bar{\Gamma}_{01}^{0}=4 m^{2} \frac{x_{1}}{r^{4}} \quad \bar{\Gamma}_{02}^{0}=4 m^{2} \frac{x_{2}}{r^{4}} \quad \bar{\Gamma}_{03}^{0}=4 m^{2} \frac{x_{3}}{r^{4}}
\end{gathered}
$$

We can find the following conclusions from the nonphysical affine connection Eqs. (36-39) : (I) not all the non-physical static Schwarzschild affine connection are positive. (II) all the non-physical affine connection have the same value. (III) after the physical decomposition, the ordinary static Schwarzschild affine connection must be revised, and some ones will be smaller than previous ones, and some others will be bigger than previous [8]. (V) the differences between revised affine connection and nonrevised ones show something interesting properties, it will bring some unfamiliar observable effects in some black holes solutions $[9,10]$. Those above conclusions have never been reported before and probably help us to find the graviton from the deep universe by the modern advanced doctors.

\section{SUMMARIZATION}

In this paper, we first review the physical decomposition of vector field and non-Abelian gauge field., i.e., $A_{m}=\hat{A}_{m}+\bar{A}_{m}$. Then we present the corresponding physical decomposition of gravitational field, i.e., $\Gamma_{\sigma \mu}^{\rho}=\bar{\Gamma}_{\sigma \mu}^{\rho}+\hat{\Gamma}_{\sigma \mu}^{\rho}$, and $\mathrm{g}_{\mu \nu}=\hat{g}_{\mu v}+\bar{g}_{\mu v}$. By using Eqs. (7-8) and Eq. (33), we revise the ordinary static Schwarzschild solution with concrete computing. The final results show that the physical decomposition of gravitational field is successful and the revised static Schwarzschild affine connection bring some amazing observable effects.

\section{ACKNOWLEDGMENT}

This work is supported by the 2014 Hubei Education Department Scientific Project No. B2014058, and 2013 Hubei university of medicine graduate start-up fund NO 2013QDJZR03.

\section{REFERENCES}

[1] X.S. Chen, X.F. L,W.M. Sun, F.Wang, and T. Gold-man, Phys. Rev. Lett. 100, 232002 (2008)

[2] X.S. Chen,W.M. Sun, X.F. L, F.Wang, and T. Gold-man, Phys. Rev. Lett. 103, 062001 (2009).

[3] LIANGWen-Feng,WuMing, LIU Hui, CHEN Xiang-Song Chin.Phys.Lett..4227, Vol.25,No.12(2008).

[4] X.S. Chen and B.C. Zhu, Physical decomposition of the gauge and gravitational fields. Phys.Rev.D.83,061501(2011).

[5] X. S. Chen and B. C. Zhu, J. Phys. Soc. Jpn. 82, (2013)083001.

[6] Zhirayr G. Avetisyan. A unified mode decomposition method for physical fields in homogeneous cosmology. arXiv:1212.2408(2012).

[7] Wenting Zhou, Hongwei Yu. Spontaneous excitation of a static multilevel atom coupled with electromagnetic vacuum fluctuations in Schwarzschild spacetime. Class. Quantum Grav. 29 (2012) 085003.

[8] Erico Tanaka, Demeter Krupka. On Metrizability of Invariant Affine Connections. IJGMMP, Vol. 9, No. 1 (2012), 1250014.

[9] Keith K. Ng, Lee Hodgkinson, Jorma Louko, Robert B. Mann, Eduardo Martin-Martinez. Unruh-DeWitt detector response along static and circular geodesic trajectories for Schwarzschild-AdS black holes. Phys. Rev. D 90, 064003 (2014).

[10] Lee Hodgkinson, Jorma Louko, Adrian C. Ottewill. Static detector and circular-geodesic detectors on the Schwarzschild black hole. Phys. Rev. D 89, 104002 (2014). 